\title{
Determination of the gamma emission probabilities of ${ }^{239} \mathrm{~Np}$
}

\author{
Shang Jian-bo a , Du li-li, Bai Tao, Dai Yi-hua, Zhong Zhen-yuan, Liu Jie, and Shi Quan-lin
}

Northwest Institute of Nuclear Technology, Xi'an, China

\begin{abstract}
Np}$ is an important nuclide as the decay daughter of ${ }^{239} \mathrm{U}$ and it decays to ${ }^{239} \mathrm{Pu}$ by emitting beta particles and gamma rays with a half life of 2.356 days. The data of the emission probabilities of its gamma-rays in the open references are consistent except for the main gamma-ray of $106.1 \mathrm{keV}$, the emission probability of which varies from $25.9 \%$ to $27.2 \%$. To verify the emission probability of $106.1 \mathrm{keV}$ gamma-ray of ${ }^{239} \mathrm{~Np}$, a N-type coaxial HPGe detector was calibrated using ${ }^{241} \mathrm{Am},{ }^{133} \mathrm{Ba},{ }^{60} \mathrm{Co},{ }^{152} \mathrm{Eu}$ and ${ }^{155} \mathrm{Eu}$ reference gamma sources to get the accurate efficiency of the $106.1 \mathrm{keV}$ gamma-ray. ${ }^{239} \mathrm{~Np}$ was purified from solution containing ${ }^{243} \mathrm{Am}$, where ${ }^{239} \mathrm{~Np}$ is the alpha decay daughter of ${ }^{243} \mathrm{Am}$. The specific activity of $239 \mathrm{~Np}$ solution was determined by a $4 \pi \beta(\mathrm{PC})-\gamma$ coincidence counting device. There were 6 gamma sources prepared to measure with the HPGe detector, and the activity of ${ }^{239} \mathrm{~Np}$ in each gamma source was calculated with the weights of the solution contained in it. The emission probability of $106.1 \mathrm{keV}$ of ${ }^{239} \mathrm{~Np}$ is measured to be $(25.4 \pm 0.3) \%$, which is consistent with $25.34 \%$, the value evaluated in 2014 .
\end{abstract}

\section{Introduction}

The neptunium isotope ${ }^{239} \mathrm{~Np}$ is a short-lived $\gamma$ and $\beta$ particle emitting radionuclide, with a half-life about 56.5 hours. It decay towards ${ }^{239} \mathrm{Pu}$ with $\beta$ and $\gamma$ particle emission. It can be produced by disintegration of ${ }^{243} \mathrm{Am}$.

${ }^{239} \mathrm{~Np}$ is of great importance as a chemical yield tracer for the radiochemical determination of ${ }^{237} \mathrm{~Np}$ in samples [1]. The absence of standardized solutions and the poor quality of the associated decay scheme data inhibited the use of ${ }^{239} \mathrm{~Np}$. For this purpose, gamma emission probabilities of ${ }^{239} \mathrm{~Np}$ were determined in this work.

\section{Comparison of ${ }^{239} \mathrm{~Np}$ half-life and decay scheme}

Table 1 shows the half-life and $\gamma$-ray emission probabilities of ${ }^{239} \mathrm{~Np}$ given by several different nuclear databases [2-5]. It can be seen that there is obvious differences between these results. In this paper, we focus on the $\gamma$-ray emission probability determination.

\section{Sample chemical separation}

The original ${ }^{243} \mathrm{Am}$ solution contains both ${ }^{243} \mathrm{Am}$ and ${ }^{239} \mathrm{~Np}$. In order to get pure ${ }^{239} \mathrm{~Np}$ solution, chemical separation should be done by the following steps:

1) Appropriately mix ${ }^{243} \mathrm{Am}$ solution and $10 \mathrm{ml} \mathrm{HNO}_{3}$ solution of $0.1 \mathrm{M}$ concentration;

2) Transfer the solution mentioned above to a CLP204 cylinder, collect the solution outflow from this cylinder which should only contains ${ }^{239} \mathrm{~Np}$, and record the time;

a e-mail: shangjianbo@nint.ac.cn
3) Add $10 \mathrm{ml} \mathrm{HNO}_{3}$ solution of $1.0 \mathrm{M}$ concentration, and collect the solution outflow which should only contains ${ }^{243} \mathrm{Am}$.

After getting a solution of ${ }^{239} \mathrm{~Np}$, six sources were prepared to determined the specific activity of ${ }^{239} \mathrm{~Np}$ solution with the coincidence method, and three sources were measured by a HPGe detector whose full-energy peak efficiency curve had already been obtained.

\section{Measurement of ${ }^{239} \mathrm{~Np}$ activity}

Measurement of specific activity of ${ }^{239} \mathrm{~Np}$ concentration was achieved by $4 \pi \beta(\mathrm{PC})-\gamma$ coincidence counting system which is a standard activity counting system. The system consists of a beta counting channel constructed from a proportional counter(PC) with $\mathrm{P} 10$ gas used as the counting medium and a gamma counting channel from a $\mathrm{NaI}$ detector.

Corrections for background, dead time and accidental coincidence were carried out using classical formulae. A linear extrapolation is usually needed in this method to estimate source activity when the counting efficiency of beta channel approaches $100 \%$. The description of linear extrapolation is as follow [6]

$$
\begin{gathered}
\frac{N_{\beta} N_{\gamma}}{N_{c}}=N_{0}\left(1+K \frac{1-\varepsilon_{\beta}}{\varepsilon_{\beta}}\right) \\
\varepsilon_{\beta}=\frac{N_{c}}{N_{r}}
\end{gathered}
$$

Where $N_{\beta}, N_{\gamma}$ and $N_{c}$ are the counting rates of the beta channel, gamma channel and coincidence channel.

Six sources with masses between $68.5 \mathrm{mg}$ and $92.7 \mathrm{mg}$ were prepared onto metalized VYNS foils. Each source was measured with linear extrapolation by varying the threshold of beta channel to change the counting efficiency.

(C) The Authors, published by EDP Sciences. This is an Open Access article distributed under the terms of the Creative Commons Attribution License 4.0 (http://creativecommons.org/licenses/by/4.0/). 
Table 1. The half-life and $\gamma$-ray emission probabilities of ${ }^{239} \mathrm{~Np}$.

\begin{tabular}{|c|c|c|c|c|}
\hline $\begin{array}{c}\text { Data } \\
\text { Base }\end{array}$ & $\begin{array}{c}\text { ENDF } \\
7.1\end{array}$ & TOI & BIPM & $\begin{array}{c}\text { Nuclear data } \\
\text { sheet }\end{array}$ \\
\hline $\mathrm{T}_{1 / 2}$ & $2.356 \mathrm{~d}$ & $2.3565 \mathrm{~d}$ & $2.356(3) \mathrm{d}$ & $2.356 \mathrm{~d}$ \\
\hline$E_{\gamma} / \mathrm{keV}$ & \multicolumn{5}{|l|}{} \\
\hline 106.123 & $26.3(10)$ & $27.2(4)$ & $25.9(3)$ & $25.34(17)$ \\
\hline 209.753 & $3.42(3)$ & $3.42(5)$ & $3.42(3)$ & $3.363(20)$ \\
\hline 228.183 & $11.14(11)$ & $10.76(18)$ & $11.32(22)$ & $10.73(9)$ \\
\hline 277.599 & $14.44(10)$ & $14.38(21)$ & $14.4(1)$ & $14.51(8)$ \\
\hline 315.88 & $1.60(2)$ & $1.60(3)$ & $1.59(1)$ & $1.600(12)$ \\
\hline 334.31 & $2.06(2)$ & $2.07(3)$ & $2.04(2)$ & $2.056(13)$ \\
\hline
\end{tabular}

Table 2. Specific activities determined by each source.

\begin{tabular}{|c|c|c|}
\hline Number & Mass/g & $\begin{array}{c}\text { Specific Activity } \\
/(\mathrm{Bq} / \mathrm{g})\end{array}$ \\
\hline 1 & 0.0685 & 31220.72 \\
\hline 2 & 0.07425 & 31438.01 \\
\hline 3 & 0.07679 & 30733.17 \\
\hline 4 & 0.09266 & 31305.74 \\
\hline 5 & 0.07643 & 31089.89 \\
\hline 6 & 0.08329 & 31196.49 \\
\hline Average & \multicolumn{2}{|c|}{31157.51} \\
\hline
\end{tabular}

Table 3. Uncertainty components in the standardization of ${ }^{239} \mathrm{~Np}$ solution.

\begin{tabular}{|l|c|}
\hline Components & Value $(\%)(k=1)$ \\
\hline $\begin{array}{l}\text { Extrapolation of efficiency and Counting } \\
\text { statistics(A) }\end{array}$ & 0.77 \\
\hline Resolving time(B) & 0.1 \\
\hline Dead time(B) & 0.12 \\
\hline Background(B) & 0.1 \\
\hline weighing(B) & 0.1 \\
\hline Half time(B) & 0.02 \\
\hline Combined $(k=1)$ & 0.80 \\
\hline
\end{tabular}

Table 2 shows the activity of each source measured by the $4 \pi \beta(\mathrm{PC})-\gamma$ coincidence system. Table 3 shows uncertainty components in the standardization of ${ }^{239} \mathrm{~Np}$ solution. The specific activity of ${ }^{239} \mathrm{~Np}$ solution at the reference time was determined to be (3.12 \pm $0.05) \times 10^{4} \mathrm{~Bq} / \mathrm{g}$. The uncertainties represent two standard deviations, coverage factor $k=2$.

\section{Gamma ray emission probabilities}

Gamma ray emission rate of ${ }^{239} \mathrm{~Np}$ was determined by a N-type coaxial HPGe detector. The detector was calibrated by ${ }^{241} \mathrm{Am},{ }^{133} \mathrm{Ba},{ }^{60} \mathrm{Co},{ }^{152} \mathrm{Eu}$ and ${ }^{155} \mathrm{Eu}$. Activity of all these sources had already determined by standardized measurement system with the relative standard uncertainties less than $1 \%$. The decay data of radioactive nuclides was selected from gamma ray decay data standards for detector calibration recommended by the IAEA in 2007. The full-energy efficiency function of the HPGe is shown in Fig. 1. The full-energy peak efficiencies of gamma-ray from decay of ${ }^{239} \mathrm{~Np}$ was obtained by intercepting the absolute full-energy peak efficiency curve also with the relative standard uncertainties less than $1 \%$ [7].

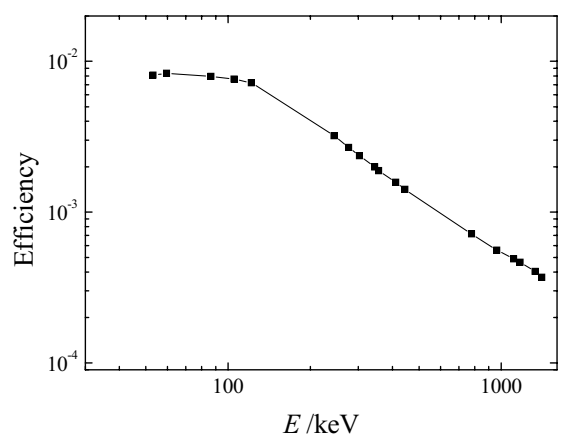

Figure 1. The full-energy efficiency function of HPGe.

Table 4. $\gamma$-ray emission probabilities and uncertain of ${ }^{239} \mathrm{~Np}$.

\begin{tabular}{|c|c|c|c|c|c|}
\hline$E / \mathrm{keV}$ & $\begin{array}{c}\gamma \text {-ray } \\
\text { emission } \\
\text { probabilities } \\
1 \%\end{array}$ & $\begin{array}{c}\text { ENDF } \\
7.1\end{array}$ & TOI & BIPM & $\begin{array}{c}\text { Nuclear } \\
\text { data } \\
\text { sheet }\end{array}$ \\
\hline 106.123 & $25.4(3)$ & $26.3(10)$ & $27.2(4)$ & $25.9(3)$ & $25.34(17)$ \\
\hline 209.753 & $3.41(6)$ & $3.42(3)$ & $3.42(5)$ & $3.42(3)$ & $3.363(20)$ \\
\hline 228.183 & $11.18(15)$ & $11.14(11)$ & $10.76(18)$ & $11.32(22)$ & $10.73(9)$ \\
\hline 277.599 & $13.84(20)$ & $14.44(10)$ & $14.38(21)$ & $14.4(1)$ & $14.51(8)$ \\
\hline 315.88 & $1.59(4)$ & $1.60(2)$ & $1.60(3)$ & $1.59(1)$ & $1.600(12)$ \\
\hline 334.31 & $2.05(6)$ & $2.06(2)$ & $2.07(3)$ & $2.04(2)$ & $2.056(13)$ \\
\hline
\end{tabular}

The gamma ray emission rate of ${ }^{239} \mathrm{~Np}$ was calculated from the gamma-ray spectrum, and then the gamma ray emission probabilities of ${ }^{239} \mathrm{~Np}$ can be determined by Eq. (3) [8].

$$
P=\frac{N_{r}}{\varepsilon_{r} A T_{L}} \frac{\lambda T_{R}}{\left(1-e^{-\lambda T_{R}}\right)} e^{\lambda T}
$$

Where $N_{r}$ is the is the full-energy peak areas of each gamma-ray, $\varepsilon_{r}$ is the full-energy efficiency of each gammaray, $A$ is the activity of ${ }^{239} \mathrm{~Np}$ at time zero, $\lambda$ is the decay constant of ${ }^{239} \mathrm{~Np}, T_{R}$ is the real time of measurement, $T_{L}$ is the live time of measurement, $T$ is the time from time zero to the beginning of measurement.

Table 4 shows emission probabilities and uncertain of main $\gamma$-ray emitted by ${ }^{239} \mathrm{~Np}$. The uncertainties represent one standard deviation, coverage factor $k=1$. Three sources were measured by HPGe to determine the $\gamma$-ray emission probabilities and each source contains about $200 \mathrm{mg}{ }^{239} \mathrm{~Np}$ solution.

\section{Conclusions}

In this work, the principal $\gamma$ emission of six energies in ${ }^{239} \mathrm{~Np}$ decay was experimentally determined as shown in Table 4. The use of absolute counting techniques to determine the activity and calibrated HPGe $\gamma$ detector has been of great importance in this work. There are still some differences between the values proposed in this work and the currently evaluated data. The emission probability of $106.1 \mathrm{keV}$ of ${ }^{239} \mathrm{~Np}$ is measured to be $(25.4 \pm 0.3) \%$, which is consistent with $25.34 \%$, the value evaluated in 2014.

\section{References}

[1] Keith-Roach M.J., Day J.P., Fifield L.K., Livens F.R., The Analyst 126, 58 (2001) 
[2] https://www-nds.iaea.org/act_ddl/data/ Np-239_com.pdf

[3] Browne E., Nuclear Data Sheets 98, 665-800 (2006)

[4] Firestone R.B., Table of Isotopes CD-ROM, California: Lawrence Berkeley National Laboratory, University of California, 1998

[5] Browne E., Tuli J.K., Nuclear Data Sheets 122, 293-376 (2014)
[6] Jia H., Zhang X., Li Q., Wang S., Fang Y., Zhao Y., Atomic Energy Science and Technology 47, 193 (2013)

[7] Bai W., Guan W., Yang J., Nuclear Electronics \& Detection Technology 35, 215 (2015)

[8] Shang J., Shi Q., Liu J., Nuclear Techniques 37, 7-12 (2014) 